\title{
PRODUCTOS DE LUJO Y DISTRIBUCIÓN A TRAVÉS DE PLATAFORMAS DE INTERNET DESDE EL DERECHO EUROPEO DE LA COMPETENCIA (TJUE C-230/16, Asunto Coty)
}

\author{
LUXURY PRODUCTS AND DISTRIBUTION THROUGH \\ INTERNET PLATFORMS FROM THE EUROPEAN \\ COMPETITION LAW \\ (ECJ C-230/16, Coty Case)
}

\author{
Alicia Arroyo Aparicio \\ Profesora Titular de Derecho Mercantil \\ UNED
}

Recibido: 15.01.2019 / Aceptado: 30.01.2019

DOI: https://doi.org/10.20318/cdt.2019.4637

\begin{abstract}
Resumen: Este estudio analiza la jurisprudencia del TJUE referida a los acuerdos de distribución selectiva de productos cosméticos de lujo y la compatibilidad de la restricción de las ventas de esos productos a través de plataformas de internet (on line) con el Derecho protector de la libre competencia de la UE. Se tiene en cuenta en particular la Sentencia de 6 de diciembre de 2017, Asunto Coty Germany GbmH c. Parfümerie Akzente $\mathrm{GmbH}$, si bien se observa la jurisprudencia en general así como desde la recaída en el Asunto Metro hasta dicha sentencia. Tres cuestiones son destacadas: distribución selectiva y Derecho de la Competencia; la compatibilidad de la prohibición de comercializar en plataformas de internet -"amazon. de" es la plataforma concreta del Asunto Coty- y la interconexión con el Derecho de marcas.

Palabras clave: distribución selectiva, plataformas de venta en internet, productos de lujo, Derecho de la Competencia, Asunto Coty.

Abstract: This study analyzes EJC Case Law referring to the selective distribution agreements of luxury cosmetic products and the compatibility of the restriction of sales of these products through online platforms, under the perspective of Antitrust European Law. In particular, the Judgment of December 6, 2017, Coty Germany GbmH c. Parfümerie Akzente GmbH is considered, but also it is important to take into account the evolution from Metro Case to Coty. Three issues are highlighted: selective distribution and Competition Law, compatibility of the prohibition to market on internet platforms - "amazon. de" was the specific platform in Coty- Case and the interconnection with the Trademarks Law.
\end{abstract}

Keywords: selective distribution, on line sales and platform bans, Antitrust Law, Coty Case.

Sumario: I. Planteamiento. 1. Asuntos Fabre y Coty. 2. Aspectos relevantes a considerar. II. Distribución selectiva y Derecho de la Competencia. III. Compatibilidad con el Derecho Antitrust de la prohibición de comercializar en plataformas de internet. IV. Interrelación con la jurisprudencia sobre marcas. 


\section{Planteamiento}

\section{Asuntos Fabre y Coty}

1. La problemática que plantean las restricciones a las ventas a través de plataformas de internet en relación con el Derecho europeo de la Competencia ha sido observada desde distintas perspectivas ${ }^{1}$ $\mathrm{y}$ ha dado lugar a interpretaciones divergentes de las Autoridades de Competencia y de los tribunales nacionales ${ }^{2}$. De manera destacada, las divergencias se pusieron de manifiesto con ocasión del pronunciamiento del TJUE en el Asunto Pierre Fabre ${ }^{3}$ en el que se consideró que "una cláusula contractual, en el marco de un sistema de distribución selectiva, que exige que las ventas de productos cosméticos $\mathrm{y}$ de higiene corporal se realicen en un espacio físico obligatoriamente en presencia de un licenciado en Farmacia, que tiene como consecuencia la prohibición de la utilización de Internet para dichas ventas, constituye una restricción por objeto" en el sentido del artículo 101.1 del TFUE. Ese pronunciamiento fue erróneamente interpretado en el sentido de considerar que la finalidad de preservar una imagen de prestigio de marca no justificaba establecer un sistema de distribución selectiva. Pero esta interpretación ha sido aclarada, junto con consideraciones sobre la problemática al inicio aludida, a finales del año 2017, el 6 de diciembre, cuando el Tribunal de Justicia se pronuncia sobre una cuestión prejudicial planteada por un tribunal alemán referido a la distribución en canales de internet -on line- de productos cosméticos de lujo (Asunto Coty) ${ }^{4}$.

Con carácter general, cabe recordar que los sistemas de distribución selectiva permiten la existencia de un comercio especializado al servicio de productos de calidad o alta tecnología ${ }^{5}$ o que preservan un estándar de lujo o prestigio ${ }^{6}$. Sin perjuicio de ello, los contratos que sustentan esos sistemas pueden ser evaluados de conformidad con el artículo 101 del TFUE y por el Reglamento 330/110 relativo a la aplicación del artículo 101, apartado 3, del TFUE a determinadas categorías de acuerdos verticales y prácticas concertadas $^{7}$ con el apoyo de las Directrices de aplicación ${ }^{8}$.

2. El resumen de los hechos del Asunto Coty puede sintetizarse como sigue. Coty Germany (en lo sucesivo, Coty) vende cosmética de lujo en Alemania y la comercialización la lleva a cabo a través de un sistema de distribución selectiva, con contratos principales y complementarios. Uno de sus distribuidores autorizados, Parfümerie Akzente (en lo sucesivo, Akzente), venía realizando la distribución en tiendas físicas y on line a través de "amazon.de". En el contrato con Akzente, estaban previstas obligaciones que Akzente debía cumplir para preservar la imagen de lujo de las marcas que cubrían los productos de Coty. Aspectos referidos en general a la decoración, la ubicación, la selección o la presentación de los productos.

En este contexto, Coty, con ocasión de la entrada en vigor del Reglamento 330/2010 relativo a acuerdos verticales ${ }^{9}$, modificó los términos del acuerdo estableciendo que las ventas en internet debían

${ }^{1}$ L. Hamelmann, J. Haucap, “Kartellrecht und Wettbewerbspolitik für Online-Plattformen“, Dice Ordnungspolitische Perspektiven, Nr. 78, Octubre 2015. Z. Georgieva, "Competition soft law in French and German courts: A challenge for ONLINE SALES BANS ONLY?”, MJECL, 2017, 24(2), PP. 175-193.

2 Tómese como ejemplo el caso de las mochilas "Deuter", acaecido en Alemania. La cuestión se concretaba en saber si el fabricante podía incluir, en un contrato con un distribuidor, una cláusula que prohibiera la venta de sus productos a través de Amazon, y otra que prohibiera el anuncio de los productos en páginas web de comparación de precios. En primera instancia, el Landgericht de Frankfurt am Main el 18 de junio de 2014 declaró que ambas prohibiciones, impuestas por el fabricante, eran anticompetitivas. Y, en segunda, el Oberlandesgericht de Frankfurt am Main de 22 de diciembre de 2015 admitió la validez de las primeras pero confirmó el carácter anticompetitivo de las segundas.

3 STJUE, 13 de octubre de 2011, C-439/09, Fabre.

${ }^{4}$ STJUE de 6 de diciembre de 2017, C-230/16, Coty Germany GmbH c. Parfümerie Akzente GMBH.

${ }^{5}$ STJCE de 25 de octubre de 1983, C-107/82, AEG-Telefunken c. Comisión.

${ }^{6}$ STJCE de 23 de abril de 2009, C-59/08, Copad.

${ }^{7}$ DOUE de 23 de abril de $2010, \mathrm{n}^{\circ} \mathrm{L} 102,1$.

${ }^{8}$ DOUE de 19 de mayo de $2010, \mathrm{n}^{\circ} \mathrm{C} 130,1$.

${ }^{9}$ En general, sobre este Reglamento: R. WISH - D. BAILEY, "Regulation 330/2010: The Commission's New Block Exemption for Vertical Agreements", CMLR, 2010, 47, Issue 6, pp. 1757-1791; y, F. Tuytschaever-f. wyjckmans, Vertical Agreements in EU Competition, $3^{\mathrm{a}}$ ed., 2018. 
realizarse a través del "escaparate electrónico" de la tienda autorizada y que se preservara el carácter de lujo de los productos. Así mismo, el nuevo acuerdo impedía que el distribuidor utilizara en el mercado on line un nombre comercial distinto o una tercera empresa que no estuviera autorizada.

De forma textual, en la STJUE ${ }^{10}$ se contiene la cláusula del contrato: "el depositario podrá ofrecer y vender los productos en Internet, a condición no obstante de que dicha actividad de venta por Internet se realice mediante un escaparate electrónico del establecimiento autorizado y que se preserve el carácter de lujo de los productos". Además -añade- "la cláusula I, apartado 1, punto 3, de dicha acta adicional prohíbe expresamente el uso de una denominación comercial diferente, así como la intervención reconocible de una tercera empresa que no sea depositario autorizado de Coty Prestige".

3. Ante la negativa a suscribir las modificaciones al contrato por parte de Akzente, Coty recurre al tribunal de primera instancia de Frankfurt para impedir que se distribuyan los productos en la plataforma on line. Este tribunal consideró que la cláusula del acuerdo era contraria bien a la Ley alemana contra las restricciones a la competencia -Gesetz gegen Wettbewerbsbeschränkungen-, bien al artículo 101 TFUE, apartado 1. Estimó en concreto que el objetivo de preservar una imagen prestigiosa de la marca no podía justificar, conforme a la sentencia de 13 de octubre de 2011, Pierre Fabre Dermo-Cosmétique ${ }^{11}$ (Asunto Fabre), la instauración de un sistema de distribución selectiva, restrictivo de la competencia por principio. La cláusula constituía también, según dicho tribunal, una restricción especialmente grave, en el sentido del artículo 4, letra c), del Reglamento 330/2010. Además, el tribunal nacional de primera instancia consideró que tampoco se cumplían los requisitos para poder acogerse a una exención individual, ni la cláusula era necesaria, porque existían otros medios adecuados pero menos restrictivos de la competencia, como la aplicación de criterios cualitativos específicos para las plataformas de terceros.

4. Coty apeló la sentencia dictada por el tribunal nacional de primera instancia ante el Tribunal Superior Regional Civil y Penal de Fráncfort del Meno, Alemania (Oberlandesgericht Frankfurt am Main). En este contexto, ese órgano jurisdiccional plantea cuestiones prejudiciales al TJUE sobre la licitud, a la luz del Derecho de la Unión en materia de Derecho de la Competencia, de los acuerdos existentes entre ambas partes del litigio. Las preguntas fueron cuatro y en su respuesta el TJUE sigue en esencia la postura del Abogado General Wahl que en suma viene a considerar que una cláusula que, a fin de preservar la imagen de los productos de lujo de una empresa, impide a un distribuidor autorizado la utilización de un sitio web de terceros no viola el Derecho de la competencia si se cumplen ciertas condiciones.

\section{Aspectos relevantes a considerar}

5. Los tres aspectos nucleares a considerar en relación con la Sentencia recaída en el Asunto Coty serían: los acuerdos de distribución selectiva, la compatibilidad de una prohibición de utilización de determinadas plataformas de internet con el Derecho europeo protector de la libre competencia, así como la interconexión entre el Derecho de marcas, la imagen de marca, con el citado Derecho. Ha de adelantarse que el Tribunal no se pronuncia sobre todos los extremos de forma desarrollada y que se han de considerar otros pronunciamientos del TJUE para analizar la relevancia del aquí destacado, en particular, los Asuntos Metro ${ }^{12}$, Copad y Fabre hasta llegar al actual.

\section{Contratos de distribución selectiva y Derecho de la Competencia}

6. Al abordar las modalidades de distribución integrada se alude a la distribución selectiva que atiende a las expectativas de los operadores de mantener un comercio especializado, sobre todo en cuanto a productos de calidad o alta tecnología, así como también a fin de preservar la imagen de lujo o

\footnotetext{
${ }^{10}$ Apartado 15 STJUE Asunto Coty.

${ }^{11}$ STJCE, 13 de octubre de 2011, C-439/09, Fabre.

${ }^{12}$ STJCE de 25 de octubre de 1977, C-26/76, Metro c. Comisión.
} 
prestigio $^{13}$ de algunos productos. Este tipo de contratos o acuerdos han de ser analizados a la luz del Derecho protector de la libre competencia en la medida en que pueden suponer efectos restrictivos sobre la competencia y han de ser evaluados bajo los criterios del artículo 101 TFUE y las normas de desarrollo, en particular, el Reglamento 330/2010 relativo a los acuerdos verticales y prácticas concertadas.

7. Los Reglamentos de exención por categorías resultan fundamentales para evaluar este tipo de contratos o acuerdos ${ }^{14}$, como también lo son las Directrices dictadas para explicar los mismos. No en vano, entre las Directrices de este Reglamento están contemplados los principales problemas referidos a la utilización de las plataformas de internet.

8. En concreto, como se recordará, en las Directrices se menciona internet como herramienta "poderosa para llegar a un mayor número y diversidad de clientes que a los que se llegaría utilizando métodos de venta más tradicionales, y por esto ciertas restricciones sobre el uso de internet se tratan como restricciones a la venta o reventa". En este contexto se consideran infracciones graves las restricciones a ventas pasivas (tener una página web se considera con carácter general venta pasiva), conteniéndose además en las Directrices algunos ejemplos concretos. Ahora bien, en ellas se afirma, por una parte, que una restricción del uso de internet por los distribuidores sería compatible con el Reglamento de Exención por Categorías de restricciones verticales en la medida en que la promoción por internet o el uso de internet llevarían a una venta activa por ejemplo en los territorios o grupos de clientes exclusivos de otros distribuidores ${ }^{15}$. Y, por otra parte, se contiene una afirmación importante a los efectos que ahora interesan en cuanto a considerar que un proveedor puede requerir niveles mínimos de calidad para el uso del sitio de internet con objeto de revender sus bienes, del mismo modo que el proveedor puede necesitar mínimos de calidad para un establecimiento o para vender por catálogo o para publicidad y promoción, en general. Y se añade que estos requisitos pueden ser pertinentes especialmente en el caso de la distribución selectiva ${ }^{16}$.

9. En este sentido, el TJUE se había pronunciado con anterioridad a propósito de que la distribución de productos de lujo requiere personas especializadas en prestar servicios específicos, en tanto que la imagen de lujo de esos productos se logra no solo por el producto en sí considerado sino también por los aspectos que rodean al mismo (se habla de "aura" o "imagen de prestigio" ${ }^{17}$ ). El Tribunal de Justicia ya consideró en el Asunto Copad que "las características y las modalidades propias de un sistema de distribución selectiva pueden, por sí mismas, preservar la calidad y asegurar el uso apropiado de dichos productos" 18 .

10. Pues bien, en el Asunto Coty el TJ, al afirmar que la distribución de productos de lujo requiere de la intervención de personas especializadas, en tanto que su imagen se logra no sólo por el producto en sí, sino por su presentación y que los sistemas de distribución selectiva pueden ser útiles a fin de "preservar su calidad y asegurar su uso apropiado" 19 , se estaría además aclarando una afirmación realizada en la Sentencia del Asunto Pierre Fabre ${ }^{20}$ que había dado pie a considerar que el objetivo de

${ }^{13}$ J. Arpio, "Sentencia del Tribunal de Justicia (Sala Primera), asunto C-230/16, Coty Germany GmbH c. Parfümerie Akzente GMBH, de 6 de diciembre de 2017 [ECLI: EU: C:2017:941], Acuerdos de distribución selectiva de productos cosméticos de lujo, restricción de las ventas a través de plataformas en línea, Ars Iuris Salmanticensis, vol. 6, Junio 2018, 329-332, esp. p. 329.

${ }^{14}$ A. Arroyo, "Reglamentos de exención", Derecho de la Competencia y Propiedad Industrial en la Unión Europea, Thomson Aranzadi, 2007, pp. 59-94.

15 Apartado 53 Directrices.

${ }_{16}$ Apartado 54 Directrices.

17 J. ARPIO, CIT., P. 330.

${ }^{18}$ STJCE de 23 de abril de 2009, as. C-59/08, Copad, apartado 28; citando a su vez a STJCE de 11 de diciembre de 1980, L'Oréal, C-31/80, Rec. p. 3775.

${ }^{19}$ Apartado 28 SJUE as. Coty.

${ }^{20}$ STJUE de 13 de octubre de 2011, as. C-439/09, Pierre Fabre Dermo-Cosmètique. Sentencia que suscitó numerosos comentarios. Entre otros: C. De FAVERI, "The Assessment of Selective Distribution Systems Post-Pierre Fabre", Global Antitrust Review 2014, pgs. 163-197; S. Enchelmaier, "Selective Distribution and the Internet: Lessons from Case C-439/09 Pierre Fabre Dermo-Cosmétique (13 October 2011), Competition on the Internet”, Surblytė G. (eds), MPI Studies on Intellectual Property and Competition Law, vol. 23, Springer, 2015. 
preservar una imagen prestigiosa de marca no podía justificar el establecimiento de un sistema de distribución selectiva. El Abogado General Wahl ${ }^{21}$ se refiere de hecho en las Conclusiones del asunto Coty a la oportunidad que tenía en este momento el TJUE de aclarar el alcance de la decisión recaída en el asunto Fabre, que había dado lugar a interpretaciones divergentes por parte de las autoridades nacionales de competencia y los tribunales.

En efecto, el TJ se preocupa en el asunto Coty de $\operatorname{aclarar}^{22}$ que la afirmación del asunto Fabre habría de ser interpretada en el contexto de dicha sentencia, siendo que en él no se dilucidaba sobre la conformidad del sistema, sino sobre una cláusula contractual. Dicha cláusula suponía una prohibición absoluta de vender en internet los productos objeto del contrato, así como habría de destacarse que se estaba ante productos cosméticos y de higiene corporal, pero no de lujo. En ese sentido, el TJ afirma ahora ${ }^{23}$ que el apartado 46 de la Sentencia de 13 de octubre de 2011, Pierre Fabre ha de entenderse en el marco de las consideraciones efectuadas por el Tribunal de Justicia para proporcionar al tribunal remitente los elementos de interpretación. Y, lo más relevante, es que el TJ en el año 2011 consideró que la necesidad de preservar la imagen de prestigio de los productos cosméticos y de higiene corporal en cuestión no constituía una exigencia que justificara una prohibición absoluta de venta de esos productos a través de internet.

11. Ahora bien, de esa afirmación del asunto Fabre no cabe deducir -afirma el TJUE en Cotyuna declaración taxativa a resultas de la cual la protección de la imagen de lujo "ya no podría justificar en lo sucesivo una restricción de la competencia, como la resultante de un sistema de distribución selectiva, respecto de todo producto -en particular, los productos de lujo-, y con ello modificar la jurisprudencia reiterada del Tribunal de Justicia" ${ }^{24}$.

12. A partir de lo hasta aquí dicho el TJ considera ${ }^{25}$ que un sistema de distribución selectiva de productos de lujo dirigido a preservar la imagen de prestigio de esos productos resulta conforme con el artículo 101, apartado 1, del TFUE siempre que: (i) los revendedores sean elegidos en virtud de criterios objetivos de carácter cualitativo, establecidos de modo uniforme respecto a todos los revendedores potenciales; y (ii) los criterios exigidos no traspasen la frontera de lo necesario.

Se recordará que en el Asunto Metro del año 1977 se asentaron lo que se ha conocido como los criterios de dicha Sentencia, que han sido seguidos en la actual Sentencia recaída en el as. Coty, ${ }^{26}$ consistentes en que un sistema de distribución selectiva no sería contrario al actual artículo 101.1 TFUE si: (i) la naturaleza del producto requiere de una distribución selectiva para preservar su calidad y asegurar su uso correcto; (ii) los revendedores sean elegidos en condiciones uniformes y de forma no discriminatoria; y (iii) que los criterios no vayan más allá de lo necesario.

13. De las condiciones o requisitos se ocupan más detalladamente las Conclusiones del Abogado General (AG) Wahl. De hecho, el AG consideró que la interpretación dada por los procedimientos nacionales en primera instancia no era adecuada en tanto que al vendedor no se le impedía desde un primer momento solicitar a sus minoristas autorizados que vendieran productos en ciertas condiciones y ubicaciones, sea en sedes físicas o en sedes electrónicas. También afirma el $\mathrm{AG}^{27}$ que la competencia de precios no es la única forma de competencia efectiva, pues "es sobre la base de esa premisa que deben considerarse los sistemas de distribución selectiva (basados en criterios cualitativos)".

\footnotetext{
${ }^{21}$ Conclusiones AG Wahl 26 de julio de 2017, as. C-230/16, Coty.

${ }^{22}$ I. Antón JuÁrez, "Los productos de lujo y su venta en Internet a través de plataformas digitales: en torno a la STJUE de 6 de diciembre de 2017, Coty Germany", Revista de Derecho de la Competencia y de la Distribución, nº 22, 1 enero de 2018, se refiere a que se "matiza" la afirmación del asunto Fabre.

${ }^{23}$ Apartado 35 STJUE as. Coty.

${ }^{24}$ STC 136/2010, de 2 de diciembre.

${ }^{25}$ Apartado 36 STJUE as. Coty.

${ }^{26}$ A. Cicala, K. Haegeman, R. Cuff, "From Metro to Coty: A story to be continued? The CJEU's Judgmen in Coty Germany GmbH v. Parfümerie Akzente GmbH”, IAR, n. 2(2017), pp. 99-111, esp. p. 100.

27 Aps. 33 y 34 Conclusiones AG asunto Coty.
} 
14. En efecto, desde la Sentencia Metro se ha reconocido la legalidad de los sistemas de distribución selectiva basados en criterios cualitativos pero objetivos (determinados de manera uniforme y aplicados de manera no discriminatoria, incluida la preservación de la imagen). Aún más, el AG añade que se ha venido aceptando de manera progresiva que los sistemas de distribución selectiva pueden incluso tener efectos positivos en la competencia: favoreciendo y protegiendo el desarrollo de la imagen de marca, especialmente en caso de artículos de lujo.

Entre marcas: "Constituyen un factor de fomento de la competencia entre los proveedores de productos de marca, a saber, la competencia intermarcas, pues permiten a los fabricantes organizar eficazmente la distribución de sus productos y satisfacer a los consumidores"28. Por otra parte se señala, en relación con la competencia intramarca en caso de distribución selectiva que podría disminuirse tanto el número de distribuidores de los productos objeto del contrato como de la competencia intramarca, en particular, en términos de precios. Si bien añade el siguiente matiz: "cuanto más severos son los criterios de selección que impone el proveedor, más se expone este, debido a la reducción de la distribución de los productos que se deriva de este hecho, a una pérdida de mercado y de clientela. A menos que disponga de un «poder de mercado» significativo, el proveedor, cabecera de red, tendrá en principio que «autorregular» su comportamiento para ajustarse a las reglas de competencia"29.

15. En el apartado 72 de sus Conclusiones el AG desarrolla la perspectiva del Derecho de Marcas, en una opinión que parece acertada. Se refiere al "aura de lujo" y "prestigio" antes referida que perciben los consumidores en los productos y derivada no sólo del aspecto material del producto en sí $^{30}$. En efecto, en el Asunto Copad, el TJUE, en el marco de un acuerdo de distribución de productos de lujo (de corsetería en el caso concreto), puso en conexión la concesión de una licencia con el ejercicio de los derechos de marca de Dior. De manera especial en cuanto a la puesta en circulación de los bienes por vez primera. En el Asunto Coty prestige ${ }^{31}$, el TJUE destacó que el carácter exclusivo del Derecho de marcas tiene como consecuencia que cada utilización de la marca sin el consentimiento del titular infringe el Derecho de marcas.

\section{Compatibilidad con el Derecho Antitrust de la prohibición de comercializar en plataformas de internet}

16. En el Asunto Coty el TJUE se plantea dar respuesta a la segunda pregunta prejudicial consistente en saber si la cláusula del contrato es o no contraria con el artículo 101, apartado 1, del TFUE. Se trata de averiguar si resulta adecuada al objetivo perseguido de preservar la imagen de lujo y de prestigio, así como si la prohibición excede de lo necesario para lograr ese objetivo.

17. En cuanto a si la cláusula del contrato se adecúa a preservar la imagen de lujo, el TJUE responde afirmativamente en la medida en que: (i) se persigue que el proveedor tenga la garantía de que los productos se vinculen exclusivamente a los distribuidores autorizados también en el marco del comercio electrónico; (ii) permite al proveedor llevar el control de que sus productos se venden en línea en un entorno que corresponde con las condiciones de calidad acordadas con sus distribuidores autorizados; y (iii) el que la venta en línea se efectúe en las tiendas de los distribuidores autorizados contribuye a la imagen de lujo que se busca lograr para los consumidores.

18. También observa el TJUE que la cláusula no va más allá de lo necesario en la medida en que la prohibición de venta en internet no es absoluta, sino que "los distribuidores autorizados pueden vender en línea los productos objeto del contrato tanto a través de sus propios sitios de Internet, si disponen

\footnotetext{
${ }^{28}$ Ap. 42 Conclusiones AG Wahl as. Coty.

${ }^{29}$ Ap. 44 Conclusiones AG Wahl as. Coty.

${ }^{30}$ STJUE de 23 de abril de 2009, C-59/08, Copad, apartados 24 a 28.

${ }^{31}$ STJUE de 3 de junio de 2010, C-127/09, Coty Prestige Lancaster Group.
} 
de un escaparate electrónico de la tienda autorizada y se preserva el carácter lujoso de los productos, como mediante plataformas de terceros no autorizadas si la intervención de éstas no es evidente para los consumidores" ${ }^{\prime 2}$. El Tribunal aporta además datos estadísticos sobre comercio electrónico ${ }^{33}$ conforme a los que el principal canal de distribución lo constituyen las tiendas en línea de los distribuidores y apunta a un dato relevante como es que, al no existir relación contractual entre el proveedor y las plataformas de terceros que permita exigir al proveedor que éstas cumplan las condiciones de calidad impuestas a sus distribuidores autorizados, "no cabe considerar que la autorización concedida a dichos distribuidores de recurrir a tales plataformas a condición de que éstas respondan a las exigencias de calidad predefinidas sea tan eficaz como la prohibición de la que se trata en el litigio principal"34.

19. El TJUE concluye así que, sin perjuicio de las comprobaciones que corresponde efectuar al órgano jurisdiccional remitente, tal prohibición resulta lícita a la luz del artículo 101 TFUE, apartado 1. Todo ello siempre que la prohibición se establezca de forma uniforme, se aplique de forma no discriminatoria y sea proporcionada al objetivo perseguido.

20. El Tribunal examina las dos últimas cuestiones -tercera y cuarta- de forma conjunta. En ellas el órgano jurisdiccional alemán preguntaba, para el supuesto de que la cláusula estuviera comprendida en el ámbito de la prohibición del artículo 101, apartado 1, y hubiere de valorarse su posible exención con arreglo al apartado 3 del mismo artículo, si la prohibición de recurrir de manera evidente a terceras empresas para las ventas por internet podría considerarse una "restricción por objeto de la clientela del minorista" o una "restricción por objeto de las ventas pasivas a los usuarios finales". Ambas restricciones son, como es conocido, consideradas graves conforme al artículo 4 del Reglamento 330/2010 de la Comisión y en virtud de cualquiera de ellas ya no cabría el beneficio de la exención por categorías ${ }^{35}$. El TJUE considera que la prohibición no supone una restricción en la medida en que no se prohíbe recurrir a internet como medio de comercialización de los productos, ni tampoco supone una restricción de ventas pasivas en tanto que los distribuidores sí que pueden realizar publicidad en plataformas de terceros, así como pueden usar motores de búsqueda en línea, de manera que los consumidores pueden acceder a la oferta en línea de los distribuidores autorizados utilizando dichos motores. Téngase en cuenta, además, que esta conclusión del TJUE estaría en consonancia con el Informe final de mayo de 2017 de la Comisión respecto del Comercio electrónico ${ }^{36}$ y que la consecuencia importante es que: una prohibición semejante no supone ni una restricción por objeto de la clientela ni una restricción de ventas pasivas.

21. En suma, es conforme al Derecho protector de la libre competencia europeo una cláusula que prohíbe a los distribuidores autorizados de un sistema de distribución selectiva de productos de lujo dirigido, con carácter principal, a preservar la imagen de lujo de dichos productos recurrir de manera evidente a plataformas de terceros para vender en Internet los productos objeto del contrato. Y del desarrollo del TJ puede deducirse ${ }^{37}$ que: (i) si se siguen las condiciones establecidas en el Asunto Metro, una prohibición que persiga preservar la imagen de lujo de un producto no violaría el artículo 101.1 del TFUE; (ii) la existencia de un sistema de distribución selectiva en sí mismo considerado no supone que se violen las normas de competencia si se cumplen las condiciones establecidas antes referidas y la naturaleza del producto requiera el uso del sistema, sin ir más allá de lo que es necesario para preservar la imagen de lujo, reconduciendo así a sus justos términos las conclusiones que podían extraerse del

\footnotetext{
32 Ap. 53 as. Coty.

${ }^{33}$ V. Informe final de 10 de mayo de 2017 resultante de la investigación en el sector del comercio electrónico de la Comisión Europea, en el que se identifican las prácticas empresariales que podrían restringir la competencia. COM(2017) 229 final.

${ }^{34}$ Ap. 56 as. Coty.

${ }^{35}$ R. Wish - D. BaILey, Competition Law, 9 ed., Oxford, 2018, esp. pg. 677.

${ }^{36}$ Apartados 41 y 42 del Informe final de 10 de mayo de 2017 sobre Comercio electrónico. Textualmente en dicho informe se refieren además a la cuestión prejudicial planteada en el Asunto Coty y se afirma lo siguiente: "sin perjuicio de la remisión prejudicial pendiente, las conclusiones de la investigación sectorial revelan que las prohibiciones (absolutas) de los mercados virtuales no se deben considerar restricciones especialmente graves con arreglo a lo establecido en el artículo 4, letras b) y c), del RECAV".

37 P. Ibañez Colomo, “Case C-230/16, Coty Germany GMbH: comon sense prevails”, Chillin Competition Blog.
} 
Asunto Fabre; y (iii), el tal vez el más relevante, una prohibición como la arriba anunciada no es una restricción grave del artículo 4 del Reglamento de restricciones verticales si no limita las ventas pasivas o los clientes a los que el distribuidor pueda dirigirse.

22. En efecto, de las tres conclusiones antes apuntadas la última implica que una prohibición de utilización de plataforma de internet como la contenida en el supuesto no sería una restricción "por objeto" en el sentido del artículo 101.1 TFUE. Y se ha considerado que de esa afirmación se deduciría que cuando se estuviera ante un supuesto similar en el que los productos fueran otros -no considerados de "lujo"-, se debería analizar caso por caso ${ }^{38}$. Ahora bien, en la Sentencia no se apunta que pueda llegarse de lo anterior a la consideración de que una prohibición similar como la contenida en el supuesto no caería dentro de la consideración de restricción grave, con independencia de la naturaleza -"lujo" o "no lujo"- del producto ${ }^{39}$. Y ello porque en la STJUE falta una aclaración o desarrollo mayor a propósito de la delimitación de los productos de lujo, puesto que por otra parte tampoco se contenía una pregunta al respecto en el planteamiento de la cuestión prejudicial.

\section{Interrelación con la jurisprudencia sobre marcas}

23. En el asunto Copad en el que el TJUE se ha basado en parte se recordará que el Tribunal sostuvo que el titular de una licencia violaría el derecho de marca si se dañaba el aura de lujo y prestigio de la marca. Y de manera similar se pronunció el TJUE en el asunto Coty Prestige antes citado ${ }^{40}$ en el que se sostenía que un uso de marca contrario al consentimiento del titular equivaldría a una violación del derecho de marca. En el asunto actual Coty el TJUE no se pronuncia respecto de los aspectos de marca de forma concreta, si bien las razones que fundamentaron las anteriores decisiones se mantienen en parte en el actual pronunciamiento.

24. Y ello porque, según el TJUE deja entrever, de forma más clara en las Conclusiones del AG Wahl, el "aura de lujo" de determinados productos queda salvaguardada no sólo por la marca sino también por las normas de competencia.

En este sentido habrían de entenderse las afirmaciones del AG al referirse a la finalidad de las normas de competencia que promueven la eficiencia económica y, en último término el bienestar de los consumidores, en tanto que no se contempla la competencia basada en los precios como único modelo posible. Aún más, se considera que existen exigencias legítimas tales como el mantenimiento de un comercio especializado capaz de prestar servicios para productos de gran calidad. ${ }^{41}$

\footnotetext{
38 P. Ibañez Colomo, cit.

39 V. opinión de P. IBAÑEz COLOMO, CIT. AL CONSIDERAR QUE SÍ QUE DEBERÍA CONCLUIRSE ASí.

40 V. parágrafo 15 supra.

41 Apartados 32 y 33 Conclusiones AG Wahl.
} 